\title{
MORTALIDADE POR NEOPLASIA MALIGNA EM RELAÇÃO A POR PROBLEMAS CARDIOVASCULARES NA POPULAÇÃ̃O FEMININA DA REGIÃO DO CARIRI CEARENSE NO ANO DE 2016
}

\author{
Daniel Gonçalves Leite ${ }^{1}$; José Maria Sousa Neto ${ }^{2}$; Julio Mariudedith Saraiva Alves Junior ${ }^{2}$; \\ Kevellyn Cruz Aguilera ${ }^{2}$; Bruna Figueiredo medeiros ${ }^{2}$; Ana Maria Lima Carneiro de Andrade ${ }^{2}$; \\ Patrícia Sobral Luna Quidute ${ }^{3}$
}

Introdução: $O$ conhecimento acerca da mortalidade por neoplasia e complicações cardiovasculares em mulheres são pontos bastante importantes a fim de que haja conscientização para o cuidado com a saúde feminina, além de proporcionar um melhor planejamento por parte do poder público das estratégias dirigidas para diagnóstico precoce e prevenção de tais agravos nessa população. Objetivo: Descrever a quantidade de óbitos gerados por Neoplasias em comparação aos gerados por complicações cardiovasculares em mulheres da região do Cariri cearense, durante o ano de 2016, a partir da casuística do Serviço de Verificação de Óbitos (SVO) de Barbalha-CE. Método: Foi realizada análise retrospectiva de 836 prontuários de pacientes examinados no SVO no período citado. Foram incluídas apenas aqueles que possuíam neoplasia ou afecções cardiovasculares (DHIP, DIC, DCBV e Outras Doenças Circulatorias) como principal causa base ou final do seu óbito. Foram incluídos no estudo apenas pacientes do sexo feminino e excluídos os menores de 20 anos (quantidades insignificantes). $\mathrm{O}$ índice de mortalidade foi dividido por faixas etárias, tendo em vista a análise de diferentes resultados para cada uma delas. Por ser o único SVO da região e por atender a sete municípios, incluindo os três mais populosos (Juazeiro do Norte, Crato e Barbalha), a casuística é representativa da população do Cariri cearense. Resultados: Ao todo 327 mulheres foram selecionadas. Na faixa de 20 a 40 anos foram verificadas $13(86.7 \%)$ mortes por problemas cardiovasculares e apenas 2(13.3\%) por neoplasias. De 41 a 60 anos foram verificadas $20(71.4 \%)$ mortes por problemas cardiovasculares e $8(28.6 \%)$ por neoplasias. Entre 61 e 80 anos foram verificados $79(85.9 \%)$ óbitos por patologias cardiovasculares e $13(14.1 \%)$ por neoplasias. Nas mulheres acima dos 80 anos foram encontrados 52(77.6\%) óbitos por complicações cardiovasculares e 15 (22.4\%) por neoplasias. Conclusão: No total, foram quantificados 202 óbitos, sendo 37 causadas por Neoplasias e 165 por patologias cardiovasculares. Esse perfil de mortalidade demonstra o grande grupo das doenças cardiovasculares ainda como principal causa de morte. Notamos também um significativo número de óbitos por neoplasias gerais, principalmente com aumento da faixa etária, demonstrando a necessidade de priorizar a prevenção, diagnóstico precoce e controle dessas doenças e de seus fatores associados para a promoção da saúde feminina no Cariri.

Palavras Chaves: Mortalidade; Neoplasias; Cardiovascular; Saúde da mulher.

\footnotetext{
1;2 Discente do curso de Medicina da Faculdade de Medicina Estácio de Juazeiro do Norte-CE;

3 Médica Radiologista. Instituto de Mama do Cariri no Juazeiro do Norte-CE;

Autor Corespondente: danielg11000@ hotmail.com.
}

16 Id on Line Rev. Mult. Psic. V.12, N. 40. 2018 - ISSN 1981-1179 EDIÇÃO ESPECIAL: I CURSO DE ONCOLOGIA DO CARIRI / II JORNADA DE PESQUISA QUANTI-QUALITATIVA EM ONCOLOGIA. JUAZEIRO DO NORTE, 05 A 10 DE MARÇO DE 2018. Edição eletrônica em http://idonline.emnuvens.com.br/id 